\title{
Evaluation of Different Local Drug Delivery Systems in the Management of Chronic Periodontitis: A Comparative Study
}

\author{
Anna Abraham ${ }^{1}$, Rekha Raghavan ${ }^{2}$, Ajesh Joseph $^{3}$, MP Shyamala Devi $^{4}$, Megha Varghese $^{5}$, PV Sreedevi $^{6}$
}

\begin{abstract}
Aim: The present study aimed to assess the use of various local drug delivery systems in the management of chronic periodontitis.

Materials and methods: A total of 60 patients aged around 30-55 years were included. The subjects who were enrolled under took a phase I therapy that included scaling and root planing (SRP). Patients who satisfied the conditions for selection to enter the trial were assigned randomly to three groups, with each group consisting of 20 participants as follows: group I: controlled-release drugs-chlorhexidine gel, group II: metronidazole gel, group III: tetracycline fibers. The plaque index (PI), gingival index (GI), and periodontal pocket depth (PPD) were recorded after 1 st week as the baseline data and were recorded again after 15 days and 30 days post-baseline.

Results: The mean GI scores were $1.32 \pm 0.10,0.88 \pm 0.16$, and $0.76 \pm 0.12$, at baseline, 15 days, and 30 days, respectively, in group I. In group II, the mean $\mathrm{Gl}$ score reduced to $1.09 \pm 0.83$ at 30 days from $1.48 \pm 0.27$ at baseline. Likewise, in group III the mean Gl score reduced to $0.90 \pm$ 0.62 at 30 days from $1.38 \pm 0.06$ at baseline. All the groups demonstrated a statistically significant difference at various intervals. The mean PI score decreased to $0.90 \pm 0.78$ at 15 days from $1.46 \pm 0.22$ at baseline in group III. A statistically significant difference at different intervals was seen in group III only. In all groups, the intergroup comparison of PPD was found to be statistically significant.

Conclusion: This study demonstrated that although thorough SRP is an effective treatment method for elimination of chronic periodontal pockets, improved results can be obtained by adjunctive use of locally administered chlorhexidine gel, metronidazole gel, and tetracycline fibers. Clinical significance: The use of the adjunctive local drug delivery system along with mechanical cleansing in the treatment of periodontal pockets in chronic periodontitis is therapeutically beneficial.

Keywords: Chronic periodontitis, Local drug delivery, Periodontal pocket, Scaling and root planing.

The Journal of Contemporary Dental Practice (2020): 10.5005/jp-journals-10024-2779
\end{abstract}

\section{INTRODUCTION}

Bacterial infection and microbial plaque start the inflammation process in the periodontal tissues. The bacterial organisms form a complex and highly organized biofilm in the periodontal pocket. With continual formation, the biofilm extends subgingivally and the patient finds it difficult to clean the deeper areas during oral hygiene maintenance. The standard treatment choice in this condition comprises of mechanical debridement that aims at removal of the subgingival microorganisms and deposits, thereby providing an even, clean, and well-matched root surface. However, the position of the lesion and the complex root anatomy may hinder the treatment and avoid adequate reduction of the bacterial load in many instances. ${ }^{1}$

The common treatment option for periodontitis is scaling and root planing (SRP). This includes elimination of supragingival and subgingival plaque and calculus, thus restoring the healthy state of oral tissues. ${ }^{2}$ Nevertheless, the treated areas may develop regrowth of microorganisms and so these days, pharmacological agents are being used along with mechanical debridement procedures. ${ }^{3}$

The complete elimination of periodontal pathogens may not be achieved by nonsurgical procedures alone due to specific nature of the bacteria that cause periodontal diseases and their position within the gingiva or areas that are deep and inaccessible. The combination of nonsurgical and surgical procedures can treat periodontal diseases. Nowadays, the combined use of antimicrobial agents and/or host-modulating agents along with mechanical debridement procedures is being tested as an effective management option for periodontal diseases. ${ }^{4}$

\footnotetext{
${ }^{1-3}$ Department of Periodontics, Educare Institute of Dental Sciences, Malappuram, Kerala, India

4,5 Department of Periodontics, PSM College of Dental Sciences and Research, Thrissur, Kerala, India

${ }^{6}$ Department of Conservative Dentistry and Endodontics, PSM College of Dental Sciences and Research, Thrissur, Kerala, India
}

Corresponding Author: Anna Abraham, Department of Periodontics, Educare Institute of Dental Sciences, Malappuram, Kerala, India, Phone: +91 9995885339, e-mail: annachally@yahoo.com

How to cite this article: Abraham A, Raghavan R, Joseph A, et al. Evaluation of Different Local Drug Delivery Systems in the Management of Chronic Periodontitis: A Comparative Study. J Contemp Dent Pract 2020;21(3):280-284.

Source of support: Nil

Conflict of interest: None

The local drug delivery system causes less drug resistance and reduced overall side effects and is associated with higher dispersion of the drug in the affected site ensuing in the complete removal of periodontal pathogens. Several agents have been used either individually or as an adjunct to SRP to stop additional progression of periodontal disease. These pharmacological agents include doxycycline, minocycline, tetracycline, metronidazole, chlorhexidine, alendronate, and simvastatin. ${ }^{5}$ The subgingival use of antimicrobial delivery system is an extensive method that includes various antimicrobial agents and several delivery systems that affect the concentration and gradual maintenance of local drugs.

() The Author(s). 2020 Open Access This article is distributed under the terms of the Creative Commons Attribution 4.0 International License (https://creativecommons. org/licenses/by-nc/4.0/), which permits unrestricted use, distribution, and non-commercial reproduction in any medium, provided you give appropriate credit to the original author(s) and the source, provide a link to the Creative Commons license, and indicate if changes were made. The Creative Commons Public Domain Dedication waiver (http://creativecommons.org/publicdomain/zero/1.0/) applies to the data made available in this article, unless otherwise stated. 
Therefore, this study was done to assess the efficacy of different local drug delivery systems in the treatment of chronic periodontitis.

\section{Materials and Methods}

The present study was conducted in the Department of Periodontics and the ethical approval for the study was obtained from the Educare Institute of Dental Sciences, Kerala. A total of 60 patients aged around $30-55$ years were included based on the following inclusion and exclusion criteria:

\section{Inclusion Criteria}

(1) Patients were with good systemic health (free from hypertension, stroke, poorly controlled diabetes, etc.); (2) two or more teeth that are not adjacent and have a persistent periodontal pocket (probing depth of at least $5 \mathrm{~mm}$ ) with bleeding or suppuration on probing (Fig. 1).

\section{Exclusion Criteria}

(1) Tooth with furcation involvement; (2) aggressive periodontitis; (3) use of antimicrobial therapy systemically 2 months before entering the study; (4) known allergy to chlorhexidine, tetracycline, or metronidazole; (5) periodontal surgeries in the past; (6) smoking; (7) periodontal treatment done $\leq 3$ months before the baseline visit.

\section{Initial Therapy}

The subjects who were enrolled undertook a phase I therapy that included SRP performed by a solo operator (P6 Piezo electric scaler, BONART, Taiwan, ROC; and GraceyCurrettes, Hu Freidy, Chicago, IL, USA), after which instructions related to oral hygiene maintenance were provided. All the patients were recalled after 1 week of phase I therapy and assessed to approve the conditions for selection. Patients who satisfied the conditions for selection to enter the trial were assigned randomly to three groups, with each group consisting of 20 participants as follows:

\section{Group I: Controlled-release Drugs: Chlorhexidine Gel (CHLO-SITE)}

Scaling and root planing was done at baseline till an even, clean, and hard surface was obtained as expected by the investigator. A syringe was used to apply chlorhexidine gel (CHLO-SITE) directly into the pocket (Fig. 2).

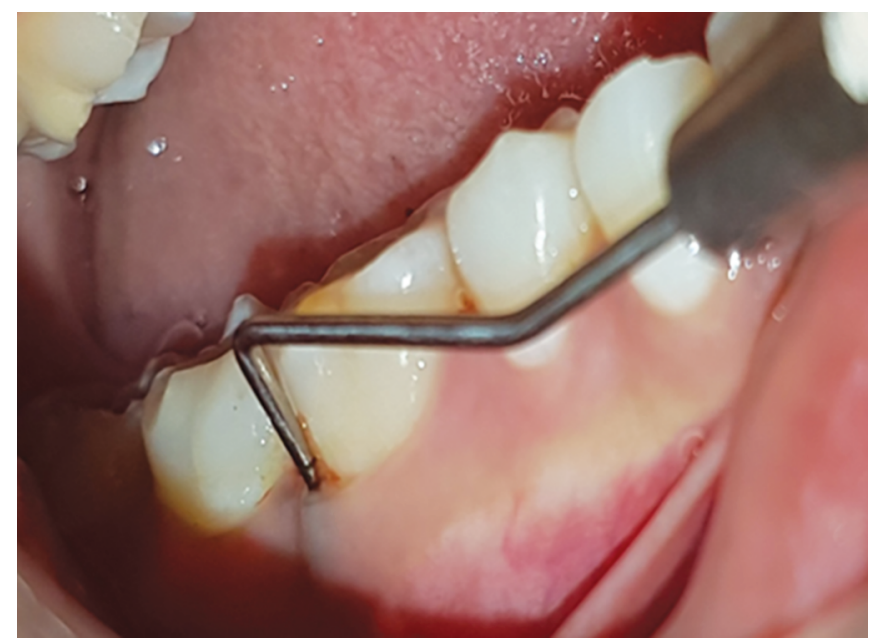

Fig. 1: Measuring probing pocket depth

\section{Group II: Metronidazole (Metrogyl) Gel}

Scaling and root planing was done at baseline till an even, clean, and hard surface was obtained as expected by the investigator. About $1 \mathrm{~mL}$ of metronidazole gel was applied subgingivally to the base of the pocket.

\section{Group III: Tetracycline Fibers (Periodontal Plus AB)}

Scaling and root planing was done at baseline till an even, clean, and hard surface was obtained as expected by the investigator. The periodontal pocket was filled with tetracycline fibers (periodontal plus AB) (Fig. 3).

At first, periodontal examination of the entire oral cavity was performed and participants satisfying inclusion criteria undertook SRP. The recall was considered as the baseline visit after 1 week. The plaque index $(\mathrm{PI})$, the gingival index $(\mathrm{GI})$, and periodontal pocket depth (PPD) were noted during baseline visit (before the local drug delivery), and these indices were rerecorded 15 and 30 days after the local drug delivery. The preliminary periodontal treatment included oral hygiene instructions $(\mathrm{OHI})$ for plaque control, which could be self-performed (such as, inter dental tooth brushing and flossing), and patient motivation was also provided.

\section{Results}

The collected data were analyzed using Statistical Package for Social Sciences (SPSS) version 20 (SPSS Inc., Chicago, IL, USA). For statistical analysis, the ANOVA test was used to establish the significance of the intergroup differences. A $p$ value of less than 0.05 was taken as statistically significant.

Table 1 and Figure 4 show the mean values of the $\mathrm{Gl}$ at baseline, 15 days, and 30 days. The mean GI scores were $1.32 \pm 0.10,0.88 \pm$ 0.16 , and $0.76 \pm 0.12$, at baseline, 15 days, and 30 days, respectively, in group I. The mean $\mathrm{Gl}$ score reduced from $1.48 \pm 0.27$ at baseline to $1.09 \pm 0.83$ at 30 days in group II. Likewise, in group III, the mean Gl score decreased from $1.38 \pm 0.06$ at baseline to $0.90 \pm 0.62$ at 30 days. All the groups demonstrated a statistically significant difference at various intervals.

The mean value for the $\mathrm{PI}$ at baseline, 15 days, and 30 days were as shown by Table 2 and Figure 5. In group I, there was a reduction in the mean PI score from $1.44 \pm 0.42$ at baseline to $1.28 \pm 0.68$ at 15 days. In group II, the mean PI score decreased from $1.38 \pm 0.09$ at baseline to $1.10 \pm 0.08$ at 30 days. At 15 days, the mean PI score

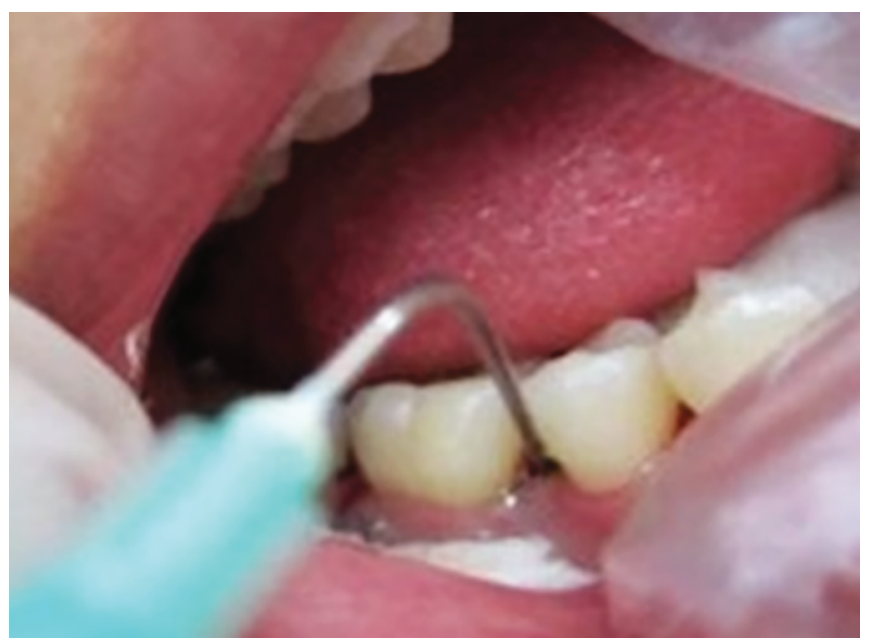

Fig. 2: Placement of chlorhexidine gel 


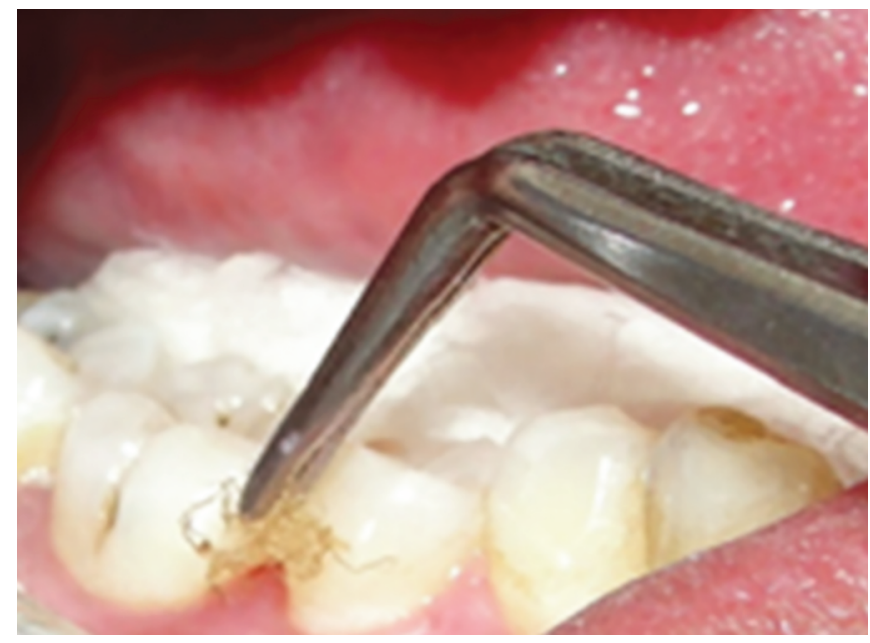

Fig. 3: Placement of tetracycline fibers

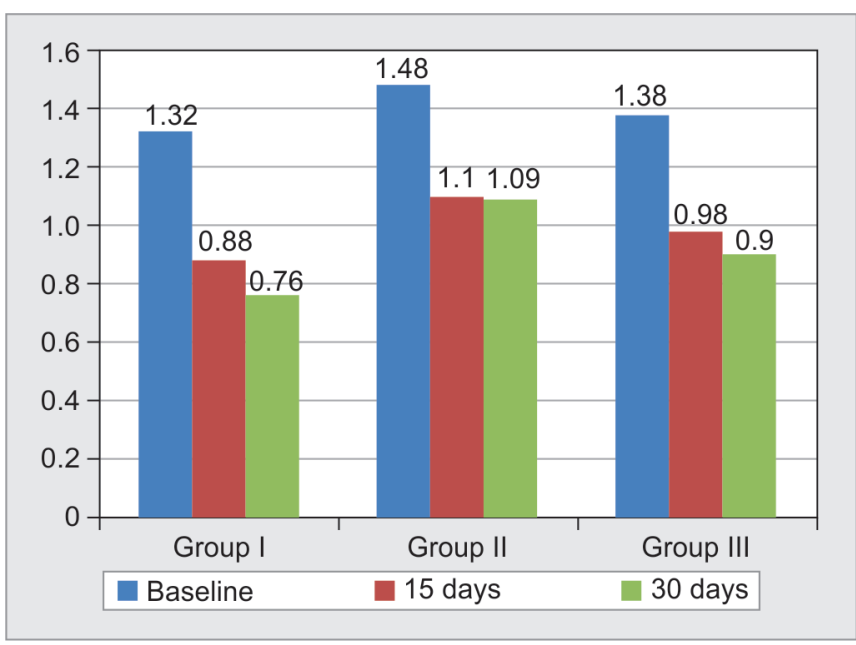

Fig. 4: Comparison of gingival index mean value at baseline, 15 days, and 30 days

Table 1: Gingival index mean value comparison at baseline, 15 days, and 30 days

\begin{tabular}{|c|c|c|c|c|c|}
\hline & Groups & & Mean $\pm S D$ & Fvalue & $p$ value \\
\hline \multirow[t]{9}{*}{ Gingival index } & Group I & Baseline & $1.32 \pm 0.10$ & 6.128 & 0.01 \\
\hline & & 15 days & $0.88 \pm 0.16$ & & \\
\hline & & 30 days & $0.76 \pm 0.12$ & & \\
\hline & Group II & Baseline & $1.48 \pm 0.27$ & 8.456 & 0.01 \\
\hline & & 15 days & $1.10 \pm 0.11$ & & \\
\hline & & 30 days & $1.09 \pm 0.83$ & & \\
\hline & Group III & Baseline & $1.38 \pm 0.06$ & 6.712 & 0.01 \\
\hline & & 15 days & $0.98 \pm 0.10$ & & \\
\hline & & 30 days & $0.90 \pm 0.62$ & & \\
\hline
\end{tabular}

Table 2: Plaque index mean value comparison at baseline, 15 days, and 30 days

\begin{tabular}{|c|c|c|c|c|c|}
\hline & Groups & & Mean $\pm S D$ & Fvalue & $p$ value \\
\hline \multirow[t]{9}{*}{ Plaque index } & Group I & Baseline & $1.44 \pm 0.42$ & 7.109 & 0.18 \\
\hline & & 15 days & $1.28 \pm 0.68$ & & \\
\hline & & 30 days & $1.31 \pm 0.12$ & & \\
\hline & Group II & Baseline & $1.38 \pm 0.09$ & 8.642 & 0.06 \\
\hline & & 15 days & $1.22 \pm 0.14$ & & \\
\hline & & 30 days & $1.10 \pm 0.08$ & & \\
\hline & Group III & Baseline & $1.46 \pm 0.22$ & 8.377 & 0.01 \\
\hline & & 15 days & $0.90 \pm 0.78$ & & \\
\hline & & 30 days & $1.02 \pm 0.01$ & & \\
\hline
\end{tabular}

decreased from $1.46 \pm 0.22$ at baseline to $0.90 \pm 0.78$ in group III. A statistically significant difference at different intervals was seen in group III only.

There was a reduction in the mean value for PPD from $5.86 \pm 0.28$ at baseline to $3.20 \pm 0.08$ at 30 days in group I. Likewise, a reduction in the mean values for PPD from $5.58 \pm 0.89$ to $3.18 \pm 0.72$ was seen in group II, and from $5.66 \pm 0.68$ to $3.12 \pm 0.30$ in group III. Table 3 and Figure 6 display a statistically significant difference in intergroup comparison of all groups for PPD.

\section{Discussion}

There is strong evidence to support that thorough SRP in combination with proper plaque control by a patient can stop periodontitis. However, there have been few instances where this approach can fail to arrest periodontitis because some microorganisms may regrow in the periodontal pockets or due to incomplete mechanical debridement. Use of adjunctive treatments becomes necessary in such clinical conditions. Several investigators support a minimal advantage of adjunctive use of locally administered antimicrobials with SRP in the management of periodontitis when compared to use of SRP alone, although the difference has frequently not been statistically significant or maximum clinically. ${ }^{6,7}$

The frequent intake of systemic antibiotics over a long period of time may lead to possible risks, such as development of resistant bacterial strains, patient incompliance, and 


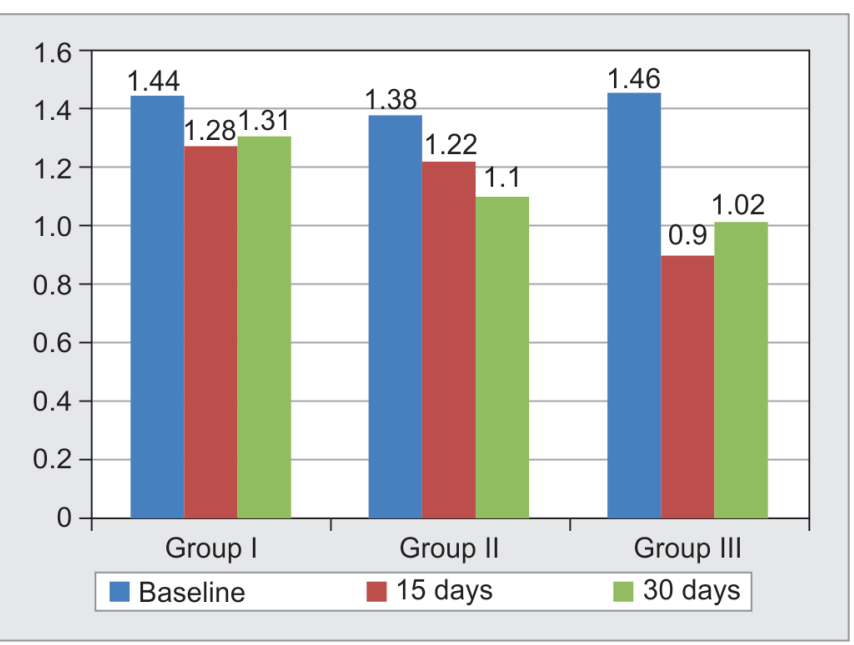

Fig. 5: Comparison of plaque index mean value at baseline, 15 days, and 30 days

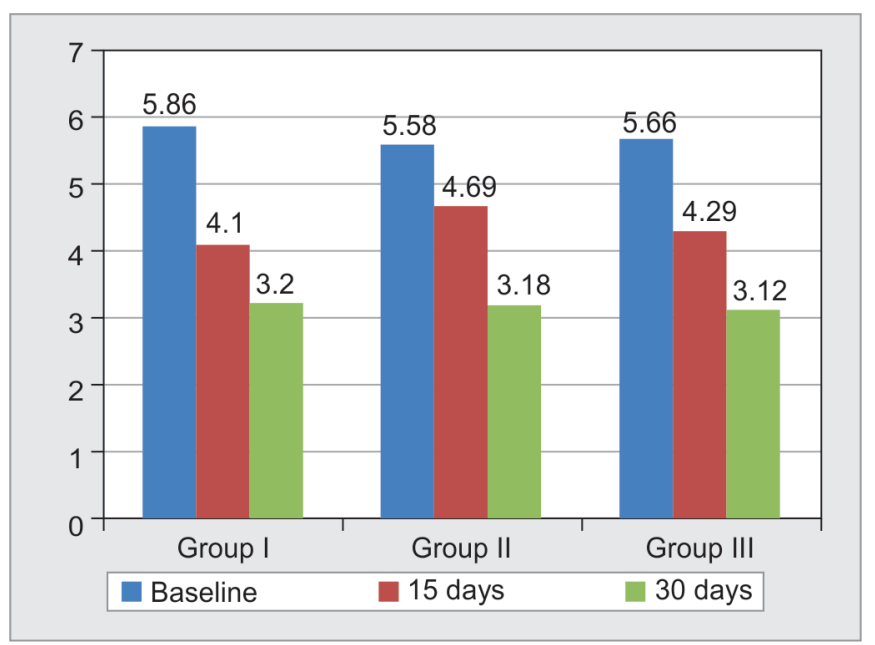

Fig. 6: Comparison of periodontal pocket depth mean value at baseline, 15 days, and 30 days

Table 3: Periodontal pocket depth mean value comparison at baseline, 15 days, and 30 days

\begin{tabular}{|c|c|c|c|c|c|}
\hline & Groups & & Mean $\pm S D$ & Fvalue & $p$ value \\
\hline \multirow{9}{*}{$\begin{array}{l}\text { Periodontal pocket } \\
\text { depth }\end{array}$} & Group I & Baseline & $5.86 \pm 0.28$ & 8.569 & 0.001 \\
\hline & & 15 days & $4.10 \pm 0.11$ & & \\
\hline & & 30 days & $3.20 \pm 0.08$ & & \\
\hline & Group II & Baseline & $5.58 \pm 0.89$ & 9.268 & 0.001 \\
\hline & & 15 days & $4.69 \pm 0.18$ & & \\
\hline & & 30 days & $3.18 \pm 0.72$ & & \\
\hline & Group III & Baseline & $5.66 \pm 0.68$ & 8.014 & 0.001 \\
\hline & & 15 days & $4.28 \pm 0.92$ & & \\
\hline & & 30 days & $3.12 \pm 0.30$ & & \\
\hline
\end{tabular}

superimposed infections. Thus, the local delivery of antimicrobials offers an important resolution to these difficulties. The increased concentrations of the drug at the target site, minimal dosage, and superior acceptance by patients and reduced number of applications are the chief advantages of the local drug delivery system. ${ }^{8}$

In our study, the mean PI score reduced significantly in the tetracycline group. Similar results were obtained by Jeong et al. ${ }^{9}$ who demonstrated a reduced supragingival plaque score in tetracycline fiber group and this could be ascribed to resolution of subgingival plaque chemically by tetracycline fibers, which could inhibit the supragingival plaque also.

We also found a statistically significant decrease in GI score in the tetracycline group. The results are same as those obtained by Soares et al. ${ }^{10}$ Reduced gingival bleeding is due to gingival inflammation control after SRP and tetracycline's known antimicrobial effect.

Over the past several years, it has been established that chlorhexidine has a beneficial effect when used as an adjunct for periodontal treatment. Chlorhexidine inhibits the microbial proteases produced by powerful periodontal pathogens and modifies the periodontal pocket's microflora. ${ }^{11}$ It may act by reducing the pellicle formation, changing the adherence of bacteria to teeth, and altering the cell wall of bacteria. The events leading to the antimicrobial effect of chlorhexidine could be an increase in permeability of the cell membrane, following which the cytoplasmic macromolecules present within the cell may coagulate. ${ }^{12}$

In the present study, the PPD reduced in group II after metronidazole treatment. An increased inflammatory response was demonstrated by Stelzel et al. ${ }^{13}$ and Mohamed Haris et al. ${ }^{14}$ in the gingival connective tissues that lie right below the junctional epithelium, and this results in a reduced number of subgingival bacteria. Griffiths et al. ${ }^{15}$ showed a significantly reduced PPD after treatment with metronidazole gel and this is in accordance with the results obtained in our study.

The PPD score reduced significantly in the tetracycline group in this study. Comparable reductions in PPD were obtained by Friesen et al. ${ }^{16}$ and Perinetti et al. ${ }^{17}$ The reductions in PPD that were obtained were due to enhanced gingival health, which may be due to decreased edematous swelling of the gingival margin and increased collagen content and this explains the decreased tissue penetrability by the probe. These findings are not same as those obtained by Drisko et al. ${ }^{18}$ who did not notice any difference.

The observations made by Banodkar and Rao ${ }^{19}$ were comparable. In addition to bacteriostatic and bactericidal activity, tetracycline benefits in periodontal treatment include dental surface adsorption and its ability to enhance attachment of fibroblasts to root surface. These results were unlike those obtained by Wilson et al. ${ }^{20}$ 
The gingival and plaque indices improved in all patients post treatment and the results were sustained during the study. The improvement in gingival and plaque indices and PPD was nearly same in all the groups. All the subjects included were based on intent to treat in this study and SRP was done at the baseline visit for complete dentition. All patients received a complete $\mathrm{OHI}$ that was provided again at every visit. These findings agree with those found by Heasman et al. ${ }^{7}$ and Jeffcoat et al. ${ }^{21}$

The short evaluation period was the major limitation of this study. The locally delivered antimicrobial agents were assessed for short-term benefit in periodontal management. The studies conducted over a long-term with follow-up period would be useful in demonstrating the actual interpretation of these results. Studies with a bigger sample size are needed in the future to assess the clinical effectiveness of these drugs as a local drug delivery system in patients with chronic periodontitis. Additionally, studies should evaluate the drug-releasing ability of these antimicrobial agents in GCF to recognize the pharmacological and molecular extent of the mechanism of action in regenerating bone in intrabony defects when applied locally.

\section{CONCLUSION}

This study demonstrated that although thorough SRP is an effective treatment method for elimination of chronic periodontal pockets, improved results can be obtained by adjunctive use of locally administered chlorhexidine gel, metronidazole gel, and tetracycline fibers.

\section{References}

1. Dodwad V, Vaish S, Mahajan A, et al. Local drug delivery in periodontics: a strategic intervention. Int J Pharm Pharm Sci 2004;4(4):30-34.

2. Greenstein G. Nonsurgical periodontal therapy in 2000: a literature review. J Am Dent Assoc 2000;131(11):1580-1592. DOI: 10.14219/jada. archive. 2000.0087.

3. Bonito AJ, Lux L, Lohr KN. Impact of local adjuncts to scaling and root planing in periodontal disease therapy: a systematic review. J Periodontol 2005;76(8):1227-1236. DOI: 10.1902/jop.2005.76.8.1227.

4. Paul TP, Emmatty R, Pulikkottil JJ, et al. Comparative evaluation of sustained release collagen device containing $5 \%$ metronidazole (metrogene) along with and without scaling and root planing at regular intervals with treatment of chronic periodontitis: a case control study. J Int Oral Health 2015;7(6):18-22.

5. Nandini N, Dodwad V, Arora K. Comparative evaluation of $1 \%$ curcumin solution and $0.2 \%$ chlorhexidine irrigation as an adjunct to scaling and root planing in management of chronic periodontitis: a clinico-microbiological study. J Pharma Biomed Sci 2012;14:1-6.

6. Reddy S, Bhowmick N, Singh S, et al. A comparison of chlorhexidine and tetracycline local drug delivery systems in management of persistent periodontal pockets - a clinical study. Int J Appl Dent Sci 2016;2(3):11-15.

7. Heasman PA, Heasman L, Stacey F, et al. Local delivery of chlorhexidine gluconate (PerioChip TM) in periodontal maintenance patients. J Clin Periodontol 2001;28(1):90-95. DOI: 10.1034/j.1600051x.2001.280114.x.

8. Slots J. Research, science and therapy committee. Systemic antibiotics in periodontics. J Periodontol 2004;75:1553-1565. DOI: 10.1902/ jop.2004.75.11.1553.

9. Jeong SN, Han SB, Lee SW, et al. Effects of tetracycline-containing gel and a mixture of tetracycline and citric acid-containing gel on non-surgical periodontal therapy. J Periodontol 1994;65(9):840-847. DOI: 10.1902/jop.1994.65.9.840.

10. Soares PB, de Menezes HH, Naves MM, et al. Effect of absorbent tetracycline-loaded membrane used in the reduction of periodontal pockets: an in vivo study. Braz Dent J 2009;20(5):414-418. DOI: 10.1590/S0103-64402009000500010.

11. Mizrak T, Güncü GN, Caglayan F, et al. Effect of a controlled-release chlorhexidine chip on clinical and microbiological parameters and prostaglandin E2 levels in gingival crevicular fluid. J Periodontol 2006;77(3):437-443. DOI: 10.1902/jop.2006.050105.

12. Anitha V, Rajesh $P$, Shanmugam M, et al. Comparative evaluation of natural curcumin and synthetic chlorhexidine in the management of chronic periodontitis as a local drug delivery: a clinical and microbiological study. Indian J Dent Res 2015;26(1):53-56. DOI: 10.4103/0970-9290.156806.

13. Stelzel M, Flores de Jacoby L. Topical metronidazole application compared with subgingival scaling. A clinical and microbiological study on recall patients. J Clin Periodontol 1996;23(1):24-29. DOI: 10.1111/j.1600-051X.1996.tb00500.x.

14. Mohamed Haris PM, Panickal DM. Role of metronidazole as a local drug delivery in the treatment of periodontitis: a review. Int J Oral Health Med Res 2017;3(6):141-145.

15. Griffiths GS, Smart GJ, Bulman JS, et al. Comparison of clinical outcomes following treatment of chronic adult periodontitis with subgingival scaling or subgingival scaling plus metronidazole gel. J Clin Periodontol 2000;27(12):910-917. DOI: 10.1034/j.1600051x.2000.027012910.x.

16. Friesen LR, Williams KB, Krause LS, et al. Controlled local delivery of tetracycline with polymer strips in the treatment of periodontitis. J Periodontol 2002;73(1):13-19. DOI: 10.1902/jop.2002.73.1.13.

17. Perinetti G,Paolantonio $M$, Cordella $C$, et al.Clinical and microbiological effects of subgingival administration of two active gels on persistent pockets of chronic periodontitis patients. J Clin Periodontol 2004;31(4):273-281. DOI: 10.1111/j.1600-051x.2004.00481.x.

18. Drisko CL, Cobb CM, Killoy WJ, et al. Evaluation of periodontal treatments using controlled-release tetracycline fibers: clinical response. J Periodontol 1995;66(8):692-699. DOI: 10.1902/ jop.1995.66.8.692.

19. Banodkar $A B$, Rao J. A comparative study of periodontal treatment using tetracycline impregnated collagen fibers as compared to scaling and root planing alone-a clinical and microbiological study. J Indian Dent Assoc 2011;5:1044-1046.

20. Wilson Jr TG, McGuire MK, Greenstein G, et al. Tetracycline fibers plus scaling and root planing versus scaling and root planing alone: similar results after 5 years. J Periodontol 1997;68(11):1029-1032. DOI: 10.1902/jop.1997.68.11.1029.

21. Jeffcoat MK, Bray KS, Ciancio SG, et al. Adjunctive use of subgingival controlled release chlorhexidine chip reduces probing depth and improves attachment level compared with scaling and root planing alone. J Periodontol 1998;69(9):989-997. DOI: 10.1902/ jop.1998.69.9.989. 